\title{
PHOTOELECTRIC CROSS-SECTIONS OF GAS AND DUST IN PROTOPLANETARY DISKS
}

\author{
T. J. Bethell and Edwin A. Bergin \\ Astronomy Department, University of Michigan, Ann Arbor, MI 48109, USA; tbethell@umich.edu \\ Received 2010 May 11; accepted 2011 July 11; published 2011 September 19
}

\begin{abstract}
We provide simple polynomial fits to the X-ray photoelectric cross-sections $(0.03 \mathrm{keV}<E<10 \mathrm{keV})$ for mixtures of gas and dust found in protoplanetary disks. Using the solar elemental abundances of Asplund et al., we treat the gas and dust components separately, facilitating the further exploration of evolutionary processes such as grain settling and gain growth. We find that blanketing due to advanced grain growth $\left(a_{\max }>1 \mu \mathrm{m}\right)$ can reduce the X-ray opacity of dust appreciably at $E_{X} \sim 1 \mathrm{keV}$, coincident with the peak of typical T Tauri X-ray spectra. However, the reduction of dust opacity by dust settling, which is known to occur in protoplanetary disks, is probably a more significant effect. The absorption of $1-10 \mathrm{keV} \mathrm{X}$-rays is dominated by gas opacity once the dust abundance has been reduced to about $1 \%$ of its diffuse interstellar value. The gas disk establishes a floor to the opacity at which point X-ray transport becomes insensitive to further dust evolution. Our choice of fitting function follows that of Morrison \& McCammon, providing a degree of backward compatibility.
\end{abstract}

Key words: opacity - protoplanetary disks - radiative transfer - stars: variables: T Tauri, Herbig Ae/Be

\section{INTRODUCTION}

The evaluation of interstellar X-ray cross-sections stretches back over four decades (e.g., Brown \& Gould 1970) and is essential for interpreting X-ray observations subject to absorption by intervening material. Today, the evaluation and manipulation of highly detailed X-ray photoelectric cross-sections for arbitrary elemental abundances is made possible by powerful publicly available codes. ${ }^{1}$ Of some historical importance is the paper by Morrison \& McCammon (1983, hereafter MM83) in which the authors present the photoelectric cross-section using a simple piecewise quadratic polynomial fitted over discrete energy intervals (spanning a total range $0.03 \mathrm{keV}<E<10 \mathrm{keV}$ ). The main benefit of using a fitting formula is that it is an expeditious way to incorporate the cross-sections into a computer code. The basic limitation of this approach is that elemental abundances become locked in. This was relaxed somewhat in the follow-up paper by Balucinska-Church \& McCammon (1992), while Wilms et al. (2000, hereafter W00) made adjustments for the $\mathrm{H}_{2}$ molecule and included more accurate estimates of elemental abundances. Both these works treated the individual elemental abundances as adjustable parameters, increasing the versatility of the results while increasing the complexity of the implementation.

Central to the piecewise construction of MM83 is the fact that-despite their low cosmic abundances relative to $\mathrm{H}$ and $\mathrm{He}$ - the metals ( $\mathrm{C}, \mathrm{O}, \mathrm{Ne}, \mathrm{Mg}$, etc.) contribute significantly to the X-ray opacity at energies above their respective $\mathrm{K}$-shell photoelectric thresholds $(0.28 \mathrm{keV}$ for $\mathrm{C}, 0.53 \mathrm{keV}$ for $\mathrm{O}$, $0.87 \mathrm{keV}$ for $\mathrm{Ne}, 1.3 \mathrm{keV}$ for $\mathrm{Mg}$, for example). These are the elements which are most readily incorporated into dust grains, although under typical interstellar conditions significant fractions of $\mathrm{C}$ and $\mathrm{O}$ (mostly in the form of $\mathrm{CO}$ ), and noble elements, persist in the gas phase. As such, the deposition of $\mathrm{X}$-ray energy $(E>0.3 \mathrm{keV})$ occurs through absorption by both gas and dust. The relative importance of gas and dust is highly energy dependent. At low energies, $E<1 \mathrm{keV}$, only a handful of metals have sufficiently low K-shell thresholds that they can

\footnotetext{
1 These codes include XSPEC (Arnaud 1996,

http://heasarc.nasa.gov/xanadu/xspec/), SHERPA (Freeman et al. 2001,

http://cxc.harvard.edu/sherpa/), and ISIS (Houck \& Denicola 2000,

http://space.mit.edu/CXC/isis/).
}

add significantly to the opacity floor set by $\mathrm{H}+\mathrm{He}$. In this case most $E \sim 1 \mathrm{keV} X$-rays will be processed by the gas. While a greater variety of elements are susceptible to K-shell ionization at higher energies $(E \sim 10 \mathrm{keV})$, the strong $\sim E^{-3}$ dependence of the photoelectric cross-section means that the total crosssection drops rapidly with increasing energy. Here heavy metals such as Fe dominate the photoelectric cross-section, implying that $10 \mathrm{keV} \mathrm{X-rays} \mathrm{will} \mathrm{likely} \mathrm{be} \mathrm{processed} \mathrm{by} \mathrm{grains.}$

It is well established observationally that young pre-mainsequence ( $\mathrm{T}$ Tauri) stars are X-ray luminous with steady $\mathrm{X}$-ray spectra typically peaking at energies of $E \sim 1-2 \mathrm{keV}$ (Feigelson et al. 2005). In addition, there is considerable variability due to the eruption of stellar flares which generate a relatively hard X-ray component, briefly extending the Xray spectrum to beyond $10 \mathrm{keV}$. In order to understand how $\mathrm{X}$-rays interact with the disk it is necessary to consider how the evolution of protoplanetary disk material might affect the total gas+dust photoelectric cross-section. In this paper, we extend the approach of MM83 by considering the effect on the X-ray photoelectric cross-section induced by two fundamental grain processes believed to occur in protoplanetary disks: dust settling and grain growth.

Interstellar dust grains become optically thick to X-rays $(E \sim 1 \mathrm{keV})$ once they grow to radii of $a \geqslant 0.5 \mu \mathrm{m}$. At this point the interior atoms will be shielded from the impinging X-rays by the layers of atoms closer to the grain surface: the interior atoms do not see the full radiation field and so do not contribute equally to the X-ray cross-section. This effect is referred to as self-blanketing (Fireman 1974; W00), rendering dust less efficient at absorbing $\mathrm{X}$-rays. This reduction in absorbing efficiency is encapsulated in the self-blanketing parameter $f_{b}(E)$. As grains continue to grow to larger sizes (the precise size is highly energy dependent) essentially all $\mathrm{X}$-rays incident upon them are absorbed (the regime of geometric optics). However, even the largest dust grains in the diffuse interstellar medium (ISM; $a_{\max } \sim 0.25 \mathrm{~mm}$; Mathis et al. 1977, hereafter MRN) are only marginally thick to $1 \mathrm{keV}$ X-rays, whereas the inferred grain-size distribution in protoplanetary disks can extend to significantly larger sizes $\left(a_{\max }>1 \mathrm{~mm}\right.$; Throop et al. 2001; Wilner et al. 2005; Lommen et al. 2009), making them strongly self-blanketing in the $E=1-10 \mathrm{keV}$ 
range. Whether large grains actually contribute to the absorption of stellar X-rays depends largely upon whether they form part of the gas-dust mixture in the X-ray irradiated surface layers.

In a steady protoplanetary disk the vertical component of the stellar gravitational field, combined with the differential drift between the ballistic grains and partially centripetally supported gas, generates friction between the gas and dust (Weidenschilling \& Cuzzi 1993). The subsequent drag forces lead to vertical settling of dust toward the disk midplane, occurring on a timescale of order $10^{5} \mathrm{yr}$. This is short compared to typical disk lifetimes $\left(>10^{6} \mathrm{yr}\right.$ ), suggesting that disks exhibit a dust-poor atmosphere overlying a thin, dust-rich midplane (Dullemond \& Dominik 2004). Following the notation of D'Alessio et al. (2006), the extent of grain removal from the upper disk layers is represented by the parameter $\epsilon$, which we simply define as the local dust-gas mass ratio relative to that in the ISM (i.e., in the ISM $\epsilon=1$ ). While dust settling occurs aggressively in typical $\mathrm{T}$ Tauri systems (the median value in Taurus is $\epsilon \sim 0.01$; Furlan et al. 2006), the modeling of infrared spectral energy distributions suggests that there is a continual replenishment and recirculation of small grains in the upper layers of the disk (Dullemond \& Dominik 2008).

In this paper, we return to the approach adopted by MM83, providing polynomial fits to the X-ray cross-sections using the most recent estimates for the solar elemental abundances provided by Asplund et al. (2009). By explicitly splitting the total X-ray cross-section into gas and dust components, it is possible to explore the effects of dust-specific processes (grain growth and settling). While the potential effect of grain growth was mentioned in the aforementioned papers, it was shown to be of little quantitative importance for grains in the diffuse ISM (typically affecting the cross-sections at the few percent level). In contrast, these effects are expected to become important in protostellar and protoplanetary systems where the nature of the gas-dust mixture departs significantly from that found in the ISM (Beckwith et al. 2000). Such results may facilitate the modeling of X-ray transport and energy deposition (Maloney et al. 1996; Igea \& Glassgold 1999; Nomura et al. 2007); resolving of the various roles of X-rays in relation to other sources of energy (e.g., cosmic-rays and ultraviolet irradiation; Glassgold et al. 2004; Ercolano et al. 2008, 2009; Owen et al. 2010); and interpreting of the observed absorption of X-rays that sample disk material (Güdel et al. 2008; Kastner et al. 2005). We present separate polynomial fits for three components.

1. A gas composed of hydrogen and helium.

2. A gas composed of hydrogen, helium, the noble gases, and some fraction of the carbon and oxygen (required to make gas-phase $\mathrm{CO}$ ).

3. Dust grains containing all the elements heavier than helium, except the noble gases and a fraction of the carbon and oxygen.

We regard Component 1 as a basal reference appropriate for chemically depleted gas. Component 2 is more typical of the gas in classical T Tauri disks. Component 3 is the dust, and it is to this that we apply our grain-specific effects. Components 2 and 3 together constitute disk material. The remainder of this paper is structured as follows. In Section 2, we describe the fitting formula and the modifications required to include the grain-specific physics. The composition of the separate gas and dust components is given in terms of the latest solar abundances. In Section 3, the resulting X-ray cross-section fits for the various components are provided both in graphical and tabulated forms. The paper concludes with Section 4 , providing a summary of the results.

\section{X-RAY CROSS-SECTION FITS}

In this section, we explicitly separate the gas and dust components and provide polynomial fits to their cross-sections. To enable backward compatibility with MM83, we have adopted the same fitting function and energy ranges. The X-ray photoelectric cross-section per $\mathrm{H}$ nucleus, $\sigma(E)$, is described by the piecewise polynomial fitting function,

$$
\sigma(E)=10^{-24} \times\left(c_{0}+c_{1} E+c_{2} E^{2}\right) E^{-3} \mathrm{~cm}^{2},
$$

where $E$ is the X-ray energy and $c_{0}, c_{1}$, and $c_{2}$ are the coefficients to be found. We extend Equation (1) by decomposing the medium into a mixture of gas and dust (e.g., Fireman 1974). Initially, we might split the total cross-section into two contributions so that $\sigma_{\text {tot }}=\sigma_{\text {gas }}+\sigma_{\text {dust }}$ (units of $\mathrm{cm}^{2}$ per H nucleus). From the point of view of computing X-ray opacities, the relatively small dust grains in the diffuse ISM can be treated as though their constituent atoms are in the gas phase (W00). In this case the distinction between gas and dust can be suspended. As discussed in Section 1, the dust in protoplanetary disks may undergo both settling and growth. To accommodate both these phenomena, we introduce two quantities, $\epsilon$ and $f_{b}(E)$, encapsulating dust settling (the physical removal of dust) and grain growth (self-blanketing), respectively. These are grain-specific parameters, and as such only affect the contribution due to dust. Therefore, we write the total cross-section as

$$
\sigma_{\text {tot }}=\sigma_{\text {gas }}+\epsilon f_{b}(E) \sigma_{\text {dust }}
$$

This is also true for combining fitting coefficients,

$$
c_{i}^{\mathrm{tot}}=c_{i}^{\mathrm{gas}}+\epsilon f_{b}(E) c_{i}^{\mathrm{dust}} .
$$

\subsection{Elemental Abundances and Composition}

The elemental abundances we have adopted are given in Table 1 (based on data from Asplund et al. 2009). It is from these abundances that we make up our total gas-dust mixture. A comparison with previous estimates of the solar elemental abundances is given in Figure 1. Although all the elements listed appear in the X-ray cross-section to some extent, carbon and oxygen (important contributors at $E \sim 1 \mathrm{keV}$ ) both have solar abundance measurements that have varied considerably over time (Asplund et al. 2005). The distribution of elements between dust and gas phases is treated in a largely binary fashion; the metals are entirely incorporated into dust $(\beta=1)$, whereas $\mathrm{H}, \mathrm{He}$, and the noble gases remain entirely in the gas phase $(\beta=0)$. The inclusion of noble elements in the gas phase is motivated not only by observations and modeling (Pascucci et al. 2007; Glassgold et al. 2007) but also by their known low chemical reactivity and low propensity for freezing-out onto grain mantles (Charnley et al. 2001). The exceptions to the simple partitioning of elements are carbon and oxygen, which are divided between the gas and solid phases according to Sofia (2004). Some C and O are necessary in the gas phase to account for observations of circumstellar CO (Calvet et al. 1991; Thi et al. 2001). The partitioning of metals between gas and dust phases is an interesting avenue for further exploration; however, we fix it in this paper to limit the number of free parameters 


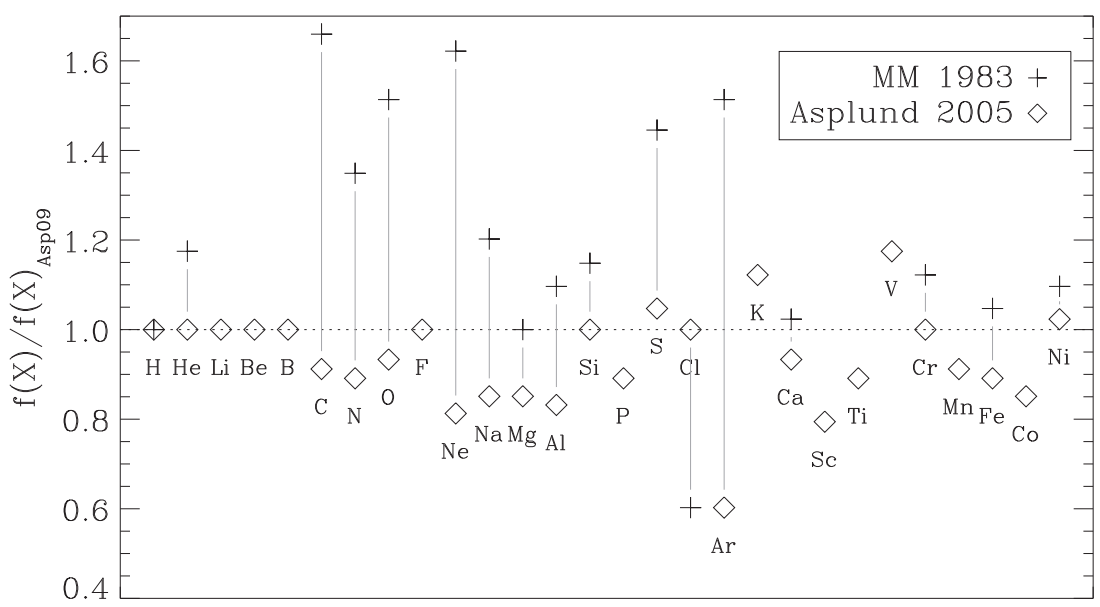

Figure 1. Comparison of the solar elemental abundances of Asplund et al. (2009) with those used previously in the literature. The quantity $f(\mathrm{X})$ is the fractional abundance of element X relative to hydrogen nuclei. Diamonds: abundances adopted by Asplund et al. (2005). Pluses: abundances adopted by MM83. Of particular note are the variations in the carbon and oxygen abundances, which over time have varied by $>50 \%$ relative to the Asplund et al. (2009) values.

Table 1

Elemental Abundances and Fraction in Dust

\begin{tabular}{|c|c|c|c|}
\hline \multirow[t]{2}{*}{ Element } & \multicolumn{2}{|c|}{ Abundance } & \multirow{2}{*}{$\begin{array}{c}\text { Dust Fraction }^{\mathrm{a}} \\
\beta_{Z}\end{array}$} \\
\hline & $12+\log A_{Z}$ & $A_{Z}=n_{Z} / n_{\mathrm{H}}$ & \\
\hline $\mathrm{H}$ & 12.0 & 1 & 0 \\
\hline $\mathrm{He}$ & 10.93 & $8.51 \times 10^{-2}$ & 0 \\
\hline $\mathrm{Li}$ & 1.05 & $1.12 \times 10^{-11}$ & 1 \\
\hline $\mathrm{Be}$ & 1.38 & $2.40 \times 10^{-11}$ & 1 \\
\hline B & 2.70 & $5.01 \times 10^{-10}$ & 1 \\
\hline $\mathrm{C}$ & 8.43 & $2.69 \times 10^{-4}$ & $0.28^{b}$ \\
\hline $\mathrm{N}$ & 7.83 & $6.76 \times 10^{-5}$ & 1 \\
\hline $\mathrm{O}$ & 8.69 & $4.89 \times 10^{-4}$ & $0.50^{\mathrm{b}}$ \\
\hline $\mathrm{F}$ & 4.56 & $3.63 \times 10^{-8}$ & 1 \\
\hline $\mathrm{Ne}$ & 7.93 & $8.51 \times 10^{-5}$ & 0 \\
\hline $\mathrm{Na}$ & 6.24 & $1.74 \times 10^{-6}$ & 1 \\
\hline $\mathrm{Mg}$ & 7.60 & $3.98 \times 10^{-5}$ & 1 \\
\hline $\mathrm{Al}$ & 6.45 & $2.81 \times 10^{-6}$ & 1 \\
\hline $\mathrm{Si}$ & 7.51 & $3.24 \times 10^{-5}$ & 1 \\
\hline $\mathrm{P}$ & 5.41 & $2.57 \times 10^{-7}$ & 1 \\
\hline $\mathrm{S}$ & 7.12 & $1.32 \times 10^{-5}$ & 1 \\
\hline $\mathrm{Cl}$ & 5.50 & $3.16 \times 10^{-7}$ & 1 \\
\hline $\mathrm{Ar}$ & 6.40 & $2.51 \times 10^{-6}$ & 0 \\
\hline $\mathrm{K}$ & 5.03 & $1.07 \times 10^{-7}$ & 1 \\
\hline $\mathrm{Ca}$ & 6.34 & $2.19 \times 10^{-6}$ & 1 \\
\hline $\mathrm{Sc}$ & 3.15 & $1.41 \times 10^{-9}$ & 1 \\
\hline $\mathrm{Ti}$ & 4.95 & $8.91 \times 10^{-8}$ & 1 \\
\hline $\mathrm{V}$ & 3.93 & $8.51 \times 10^{-9}$ & 1 \\
\hline $\mathrm{Cr}$ & 5.64 & $4.37 \times 10^{-7}$ & 1 \\
\hline $\mathrm{Mn}$ & 5.43 & $2.69 \times 10^{-7}$ & 1 \\
\hline $\mathrm{Fe}$ & 7.50 & $3.16 \times 10^{-5}$ & 1 \\
\hline $\mathrm{Co}$ & 4.99 & $9.77 \times 10^{-8}$ & 1 \\
\hline $\mathrm{Ni}$ & 6.22 & $1.66 \times 10^{-6}$ & 1 \\
\hline
\end{tabular}

Notes.

a The fraction of element $Z$ in grains.

b Sofia (2004).

(cf. Balucinska-Church \& McCammon 1992). The total crosssection of a mixture of elements ( $\mathrm{cm}^{2}$ per $\mathrm{H}$ nucleus) is found by combining the abundances with the atomic X-ray cross-sections $\sigma_{Z}$ from the online NIST database ${ }^{2}$ (Chantler 2000),

$$
\sigma=\Sigma_{Z} \sigma_{Z} A_{Z}
$$

\footnotetext{
2 http://www.nist.gov/physlab/data
}

In this paper, we only consider neutral species, requiring that the ionization state of the gas is low. In order to produce simple fits to the cross-section, we neglect solid-state features near photoelectric thresholds. The detailed study of X-ray fine structure may provide a useful diagnostic of the composition of solid interstellar material (Lee et al. 2009). As noted in W00, $\mathrm{H}_{2}$ has a cross-section that is approximately $40 \%$ larger (per $\mathrm{H}$ nucleus) than atomic $\mathrm{H}$. We adopt the enhanced $\mathrm{H}_{2}$ cross-section and assume the gas is fully molecular (i.e., $n\left(\mathrm{H}_{2}\right) / n_{\mathrm{H}}=0.5$ ). Relative to earlier work focusing on the largely atomic ISM, the inclusion of the molecular enhancement increases the crosssection most significantly at extreme ultraviolet wavelengths where hydrogen opacity dominates.

\section{RESULTS}

The well-tested IDL fitting routines POLY_FIT and SVD were used to evaluate and test the uniqueness of the fits. The fitting coefficients are given in Table 2 . The resulting opacity curves are shown in Figure 2. By comparison with a gas of pure $\mathrm{H}$ and $\mathrm{He}$, Figure 2 clearly shows that $\mathrm{C}, \mathrm{O}$, and noble gases, $\mathrm{Ne}$ and $\mathrm{Ar}$, contribute significantly to the gas opacity at energies $E>0.3 \mathrm{keV}$. Away from photoelectric edges the fitting errors are typically at the $1 \%$ level, which is small compared to the compositional uncertainties of gas and dust. Noticeable in the dust component are significant $(\sim 10 \%)$ error spikes near the metal K-edge thresholds. These errors (consistent with those in W00) arise from the numerical discretization of the elemental cross-sections, and from the fundamental limitations of a loworder fit. It is important to note that striving for a more accurate polynomial fit at K-shell edges is not entirely meaningful unless we include actual solid-state effects, which in general are too complex for a low-order polynomial fit (Draine 2003).

\subsection{Grain Growth, $f_{b}(E)$}

The derivation of the self-blanketing factor $f_{b}(E)$ for spherical, homogeneous grains is described in the Appendix. Plots of $f_{b}(E)$ versus X-ray energy for a range of grain sizes are shown in Figure 3. As a function of energy it bears the imprint of $\sigma_{\text {dust }}$ since $f_{b}(E)$ depends upon the optical depth of the grain. At low energies ( $E \ll 1 \mathrm{keV}$ ), the self-blanketing effect can be very strong (the grains are optically thick, $f_{b}(E) \ll 1$ ), but it is not seen in the total (gas+dust) opacity because at 


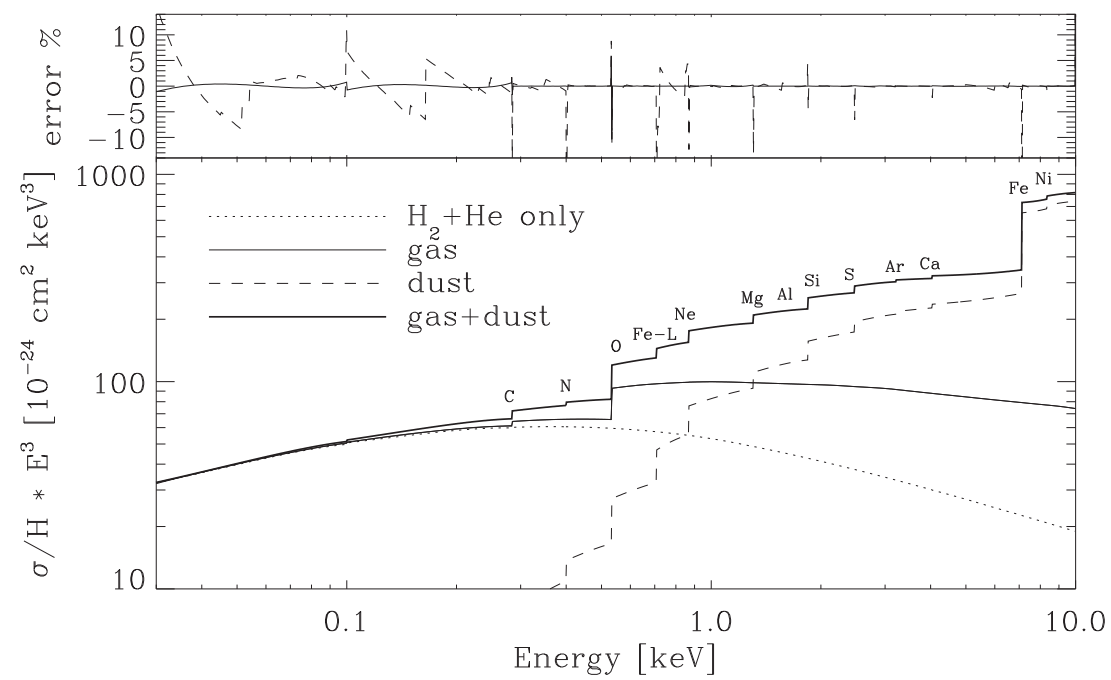

Figure 2. Bottom: X-ray cross-section of our basic gas-dust mixture using Asplund et al. (2009) solar abundances. The parametric fitting coefficients for these curves are given in Table 2. The fiducial dust contribution is calculated without modification by grain processes (i.e., $\epsilon=f_{b}(E)=1$ ). To include the effects of settling and self-blanketing one must combine the gas and dust components following Equations (2) and (3). The reader should note the $E^{3}$ scaling - the total cross-section in fact drops with increasing energy. Top: residuals of our fitting functions for gas and dust components.

Table 2

Fitting Coefficients for Different Components

\begin{tabular}{|c|c|c|c|c|c|c|c|c|c|}
\hline \multirow{2}{*}{$\begin{array}{l}\text { Energy Range } \\
(\mathrm{keV})\end{array}$} & \multicolumn{3}{|c|}{$\mathrm{H}_{2}+\mathrm{He}$} & \multicolumn{3}{|c|}{ Gas } & \multicolumn{3}{|c|}{ Dust } \\
\hline & $c_{0}$ & $c_{1}$ & $c_{2}$ & $c_{0}$ & $c_{1}$ & $c_{2}$ & $c_{0}$ & $c_{1}$ & $c_{2}$ \\
\hline $0.030-0.055$ & 14.3 & 722 & -4190 & 14.2 & 727 & -4130 & 0.0344 & -1.62 & 88.2 \\
\hline $0.055-0.100$ & 22.3 & 432 & -1530 & 22 & 445 & -1550 & -0.147 & 4.19 & 48.1 \\
\hline $0.100-0.165$ & 31.5 & 246 & -578 & 31 & 263 & -614 & -0.677 & 14.9 & 9.6 \\
\hline $0.165-0.284$ & 42 & 120 & -198 & 43.7 & 112 & -165 & -1.12 & 23.6 & -16.2 \\
\hline $0.284-0.400$ & 51.5 & 50 & -68.7 & 49 & 86 & -103 & 0.188 & 24.6 & -1.09 \\
\hline $0.400-0.532$ & 58.1 & 16.8 & -26.4 & 58.6 & 36.9 & -39.9 & -3.57 & 55.5 & -37.9 \\
\hline $0.532-0.708$ & 63.2 & -2.37 & -8.44 & 48 & 130 & -82.2 & -8.24 & 89.6 & -48.1 \\
\hline $0.708-0.867$ & 66.8 & -12.5 & -1.18 & 77.4 & 46.3 & -22 & 57.1 & -49.9 & 52.1 \\
\hline $0.867-1.303$ & 69.4 & -17.5 & 1.2 & 80.1 & 69.8 & -28.3 & 9.11 & 72.7 & -20.8 \\
\hline $1.303-1.840$ & 71.6 & -22.3 & 3.56 & 117 & 7.43 & -1.87 & -8.71 & 106 & -25.7 \\
\hline $1.840-2.471$ & 66.6 & -16.8 & 2.06 & 107 & 16 & -3.75 & 34.9 & 72.4 & -11.4 \\
\hline $2.471-3.210$ & 61.4 & -12.6 & 1.21 & 106 & 13.6 & -2.63 & 23.6 & 85.1 & -11.3 \\
\hline $3.210-4.038$ & 56.6 & -9.6 & 0.75 & 138 & -1.99 & -0.179 & 116 & 28.2 & -2.55 \\
\hline $4.038-7.111$ & 48.4 & -5.8 & 0.308 & 142 & -4.7 & 0.239 & 191 & -2.92 & 1.09 \\
\hline $7.111-8.331$ & 40.5 & -3.43 & 0.128 & 138 & -3.36 & 0.133 & 812 & -74.7 & 6.49 \\
\hline $8.331-10.00$ & 37.8 & -2.8 & 0.091 & 88.9 & 8.15 & -0.547 & -33 & 137 & -6.39 \\
\hline
\end{tabular}

Notes. The coefficients belong to the fitting function given by Equation (1) and can be combined according to Equation (3). The gas component consists of $\mathrm{H}, \mathrm{He}, \mathrm{Ar}, \mathrm{Ne}$, and a fraction $\left(1-\beta_{Z}\right)$ of $\mathrm{O}$ and $\mathrm{C}$ (see Table 1). The dust component consists of the remaining elements. The $\mathrm{H}+\mathrm{He}$ case is meant to serve as a basal reference.

these energies the total opacity is dominated by gas, not dust. At energies $E>1 \mathrm{keV}$ the metals in dust grains dominate the total (gas+dust) opacity but tend to be optically thin (for $a<100 \mu \mathrm{m})$, and as a result the self-blanketing effect is weak. At intermediate energies ( $E \sim 1 \mathrm{keV}$ ), the self-blanketing factor departs from unity once grains have grown to $a \sim 0.5 \mu \mathrm{m}$. Grains as large as $a \sim 1 \mathrm{~mm}$ are extremely optically thick, hiding $99.9 \%$ of their constituent atoms from the ambient X-rays. The grain-size distribution in protoplanetary disks is largely unknown, although the process of coagulation will tend to drive mass into larger grains. The ensemble-averaged self-blanketing factor, $\left\langle f_{b}(E)\right\rangle$, for a population of grains with a power-law size distribution, $d n / d a \propto a^{-p}$, is given by the average,

$$
\left\langle f_{b}(E)\right\rangle=\frac{\int_{a_{\min }}^{a_{\max }} f_{b}(E) a^{3-p} d a}{\int_{a_{\min }}^{a_{\max }} a^{3-p} d a},
$$

where $p=3.5, a_{\min } \sim 0.01 \mu \mathrm{m}$, and $a_{\max } \sim 0.25 \mu \mathrm{m}$ for the classic MRN distribution. The mass per decade of grain size in the MRN distribution is weakly dominated by the largest grains. This becomes increasingly true if we flatten the distribution through grain coagulation (taking mass from the small grains and placing it in the big grains). It is therefore of interest to briefly study the effects of changing the maximum grain size $a_{\max }$ and power-law index $p$. In Figure 4, we preserve the slope of the MRN distribution but allude to grain growth by gradually increasing $a_{\max }$ from the interstellar value $\sim 0.25 \mu \mathrm{m}$ to $1 \mathrm{~mm}$. The increase in $a_{\max }$ draws an increasing large fraction of the dust mass into the realm of self-blanketing. A similar effect can be achieved by reducing the power-law index $p$, as shown in Figure 5. In this case the evaluation of $\left\langle f_{b}(E)\right\rangle$ is increasingly dominated by grains with sizes $a \sim a_{\max }$ (we hold $a_{\max }=10 \mu \mathrm{m}$ so that the curves for $p<2$ can be compared 


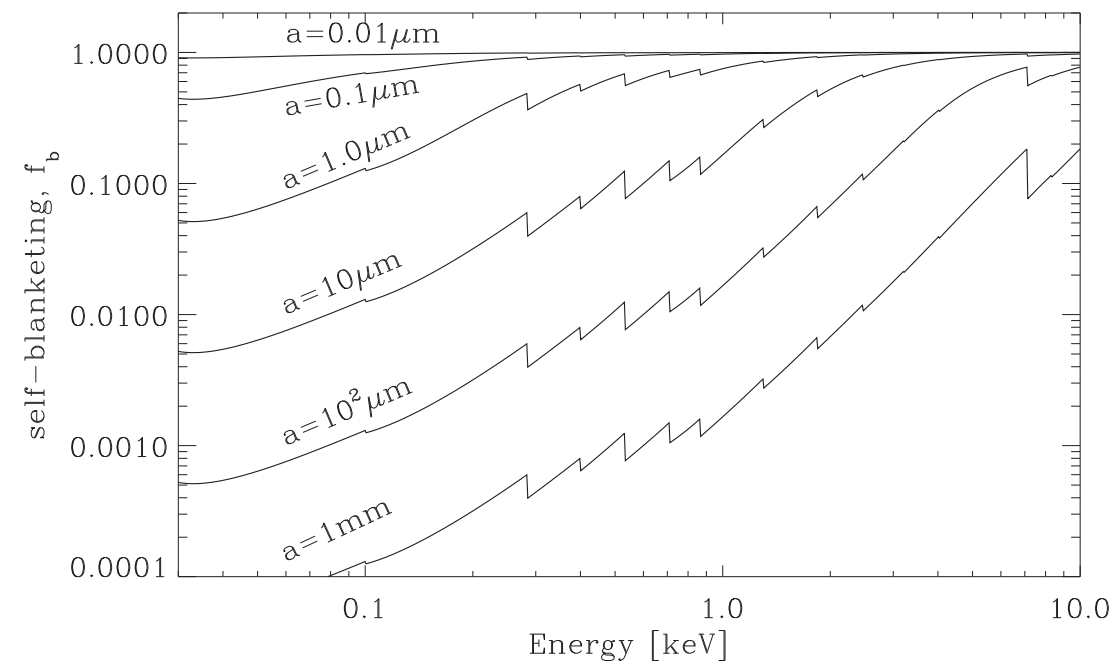

Figure 3. Self-blanketing factor for spherical dust grains of radius $a$. At $E_{X}=1 \mathrm{keV}$ a dust grain with $a \sim 0.5 \mu \mathrm{m}$ has a diametrical optical depth of $\tau \sim 1$, marking the onset of appreciable self-blanketing.

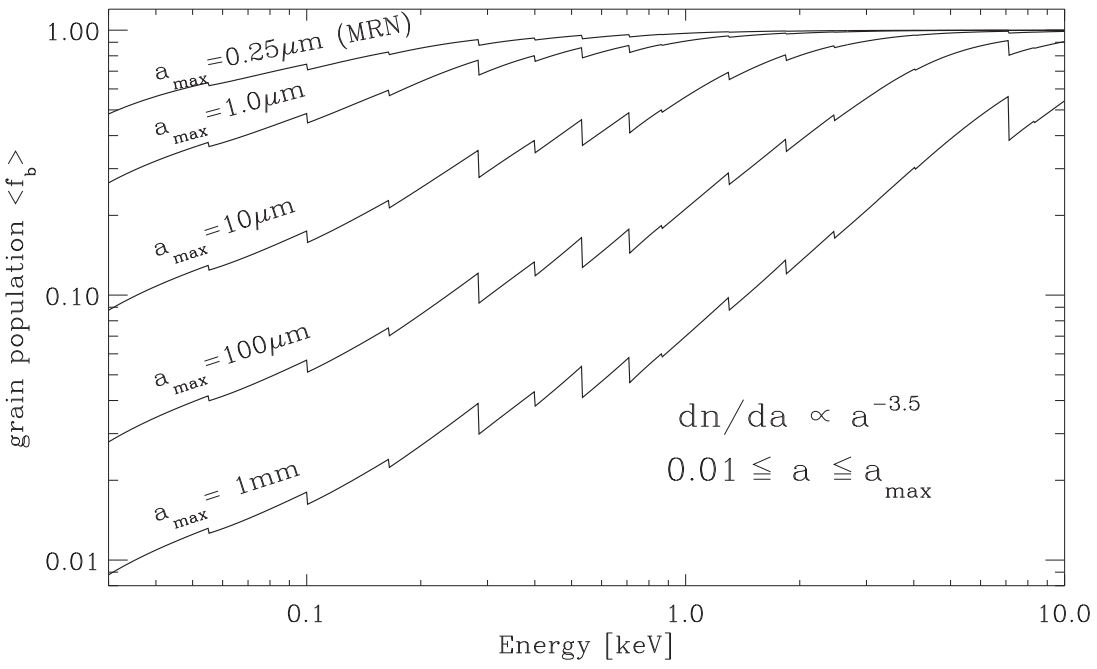

Figure 4. Self-blanketing factor averaged over a power-law size distribution of grains. The different lines correspond to changing the maximum grain size in the population, $a_{\max }$, while keeping the minimum size fixed, $a_{\min }=0.01 \mu \mathrm{m}$. The power-law index of $p=3.5$ and $a_{\max }=0.25 \mu \mathrm{m}$ is consistent with the classic MRN result for diffuse interstellar dust.

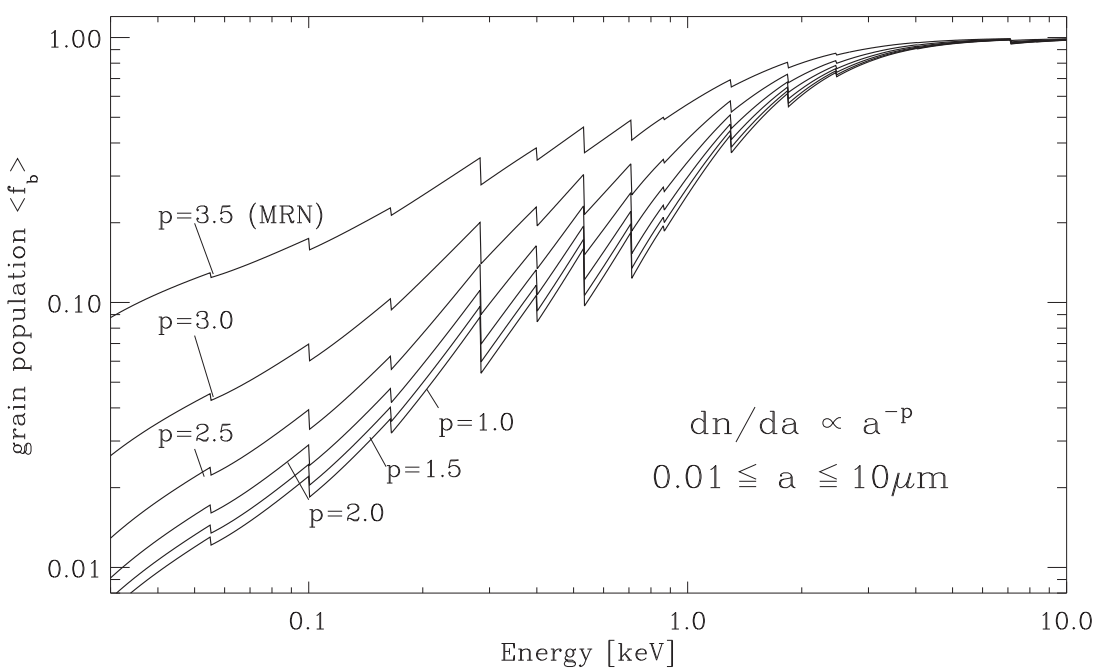

Figure 5. Self-blanketing factor averaged over a power-law MRN size distribution of grains. The different lines correspond to changing the power-law index of the population, $p$, while keeping the minimum and maximum grain sizes fixed at $a_{\min }=0.01 \mu \mathrm{m}$ and $a_{\max }=10 \mu \mathrm{m}$, respectively. The power-law index of $p=3.5$ is the classic MRN result for diffuse interstellar dust. We have extended $a_{\max }$ to a value larger than that specified by MRN, since some grain growth is required before self-blanketing manifests itself. 
Table 3

Fitting Coefficients for Dust Component Subject to Grain Growth

\begin{tabular}{|c|c|c|c|c|c|c|c|c|c|c|c|c|}
\hline \multirow{2}{*}{$\begin{array}{l}\text { Energy Range } \\
(\mathrm{keV})\end{array}$} & \multicolumn{3}{|c|}{$a_{\max }=1 \mu \mathrm{m}$} & \multicolumn{3}{|c|}{$a_{\max }=10 \mu \mathrm{m}$} & \multicolumn{3}{|c|}{$a_{\max }=100 \mu \mathrm{m}$} & \multicolumn{3}{|c|}{$a_{\max }=1000 \mu \mathrm{m}$} \\
\hline & $c_{0}$ & $c_{1}$ & $c_{2}$ & $c_{0}$ & $c_{1}$ & $c_{2}$ & $c_{0}$ & $c_{1}$ & $c_{2}$ & $c_{0}$ & $c_{1}$ & $c_{2}$ \\
\hline $0.030-0.055$ & 0.0203 & -1.5 & 47 & 0.00785 & -0.576 & 17 & 0.00260 & -0.19 & 5.52 & 0.000829 & -0.0607 & 1.75 \\
\hline $0.055-0.100$ & 0.0192 & -1.64 & 50.9 & 0.0166 & -0.918 & 20.6 & 0.00616 & -0.325 & 6.9 & 0.00203 & -0.106 & 2.21 \\
\hline $0.100-0.165$ & -0.173 & 1.69 & 40.7 & 0.00492 & -0.794 & 21.7 & 0.00703 & -0.371 & 7.65 & 0.00275 & -0.129 & 2.48 \\
\hline $0.165-0.284$ & -1.36 & 15.8 & 2.1 & -0.236 & 2.05 & 14.4 & -0.0475 & 0.28 & 6.03 & -0.0124 & 0.0528 & 2.04 \\
\hline $0.284-0.400$ & -2.96 & 27.5 & -0.943 & -0.61 & 4.29 & 16.7 & -0.103 & 0.539 & 7.52 & -0.0238 & 0.091 & 2.58 \\
\hline $0.532-0.708$ & -19.2 & 100 & -48.7 & -7.71 & 31.9 & 0.587 & -1.8 & 7.03 & 4.67 & -0.506 & 1.92 & 1.9 \\
\hline $0.708-0.867$ & 17 & 19.2 & 17.5 & 2.13 & 8.01 & 23.4 & 1.92 & -2.27 & 13 & 0.725 & -1.17 & 4.65 \\
\hline $0.867-1.303$ & -14 & 98.2 & -28.7 & -25.5 & 71.1 & -11.5 & -5.4 & 14.6 & 3.75 & -1.4 & 3.74 & 1.93 \\
\hline $1.303-1.840$ & -27.1 & 120 & -28.6 & -63 & 123 & -24.5 & -17.8 & 31.3 & -0.219 & -4.69 & 8.05 & 1.04 \\
\hline $1.840-2.471$ & 15.8 & 84.1 & -13.4 & -59.6 & 119 & -17.9 & -28 & 40.5 & -0.463 & -6.39 & 9.35 & 1.39 \\
\hline $2.471-3.210$ & 10.1 & 91.3 & -12.1 & -63.7 & 121 & -15.4 & -63.4 & 66.4 & -4.23 & -14.7 & 15.2 & 0.66 \\
\hline $3.210-4.038$ & 106 & 31.9 & -2.95 & 40.9 & 56.7 & -5.52 & -71.8 & 72.3 & -5.27 & -15.4 & 15.7 & 0.556 \\
\hline $7.111-8.331$ & 800 & -72.4 & 6.37 & 704 & -53.9 & 5.39 & 183 & 37.1 & 0.832 & 24 & 13.7 & 2.21 \\
\hline $8.331-10.00$ & -39.5 & 138 & -6.42 & -90.7 & 145 & -6.69 & -397 & 181 & -7.76 & -276 & 86 & -2.05 \\
\hline
\end{tabular}

Notes. The coefficients apply only to the dust component. $a_{\max }$ refers to the maximum grain size in an MRN grain-size distribution. The total dust mass is held constant.

to the curve for the single grain-size population $a=10 \mu \mathrm{m}$ in Figure 3). Fitting coefficients for the dust component computed using MRN distributions $\left(a_{\max }=1,10,100\right.$, and $\left.1000 \mu \mathrm{m}\right)$ are given in Table 3 .

The effect of self-blanketing on the total (gas+dust) photoelectric cross-section is shown in the bottom panel of Figure 6. The onset of grain growth initially affects the cross-section near $E_{X} \sim 1 \mathrm{keV}$ - coincident with the peak X-ray emission from $\mathrm{T}$ Tauri systems.

\subsection{Dust Settling, $\epsilon$}

The effect of dust settling is shown in the upper panel of Figure 6 and is straightforward to interpret. To isolate this effect, we show its effect applied to the standard interstellar MRN grain-size distribution, for which self-blanketing is mild due to the preponderance of small grains $(a<0.5 \mu \mathrm{m})$. Once $\epsilon$ has dropped to below 0.1 (consistent with $\mathrm{T}$ Tauri systems in Taurus) the contribution made by dust to the total opacity at $E=1 \mathrm{keV}$ is small compared to that of the gas. The gas sets a basal floor to the cross-section. Further reduction of the cross-section is only possible by depleting gas phase metals and noble elements.

\subsection{X-Ray Scattering}

The primary purpose of this paper is to quantify the photoelectric absorption cross-sections that determine how X-ray energy is deposited into gas and dust. The more general consideration of how $\mathrm{X}$-rays are transported through space requires the inclusion of scattering. Restricting the discussion to scattering by spheres of radius $a$, the main parameters that determine scattering efficiencies are the size parameter $x \equiv 2 \pi a / \lambda$ where $\lambda$ is the X-ray wavelength, and the real part of the complex refractive index, $m$. van de Hulst (1957) provides an authoritative discussion of scattering processes throughout this parameter space (see Figure 20, Chap. 10). While Mie theory provides a rigorous way to compute the extinction of individual particles, a number of approximations are applicable at the limits of this parameter space. The region of interest is reduced somewhat since $m-1 \ll 1$ for most materials (including interstellar dust candidates) at X-ray wavelengths ( $E>0.1 \mathrm{keV}$; Draine 2003). A refractive index close to unity implies that the phase of an $\mathrm{X}$-ray inside the grain only slowly deviates from that of an exterior X-ray. When the total differential phase change $2 x(m-1)$ is smaller than unity (true for most interstellar grains), one may use the Rayleigh-Gans approximation to compute the scattering efficiency (Mathis \& Lee 1991). The Rayleigh-Gans approximation asserts that the grain can be viewed as an ensemble of Rayleigh scattering centers (Overbeck 1965). The sum total of these fields gives a scattered field that is sharply peaked in the forward direction: the property responsible for the narrow halos observed around X-ray point sources (Rolf 1983; Predehl \& Schmitt 1995; Smith \& Dwek 1998). When computed for the Weingartner \& Draine (2001) grain population, 90\% of $1 \mathrm{keV} \mathrm{X}$-rays are scattered by less than $1^{\circ}$ away from the forward direction. ${ }^{3}$

In Figure 7, we show alongside the photoelectric absorption cross-section the scattering cross-sections of both gas and dust computed by Draine (2003). When the $E^{3}$ factor has been taken into account it can be seen that the non-relativistic (Thomson) scattering by gas is essentially wavelength independent. Thomson scattering - the interaction of a photon with a free (or weakly bound) electron-is due almost entirely to $\mathrm{H}$ and He since these elements carry the majority of the electrons (Igea \& Glassgold 1999). It only contributes significantly to the total extinction at $E \geqslant 6 \mathrm{keV}$. Even in dust depleted scenarios, scattering by gas is only ever important for the highest energy photons observed in $\mathrm{T}$ Tauri spectra. In contrast, dust scattering contributes significantly at lower energies, $E \sim 1 \mathrm{keV}$. However, while small-angle scattering by grains is important for interpreting observations of X-ray halos, the very narrow phase function associated with dust scattering suggests that multiple scattering events are required to produce a significant diffuse $\mathrm{X}$-ray field inside a protoplanetary disk. Thus, while the dust scattering cross-section may be large in magnitude it does not redirect radiation effectively. Since the total photoelectric ab-

\footnotetext{
3 In the limit of geometrical optics (large grain sizes, $2 x(m-1) \gg 1$ ) the absorption and scattering (diffraction) cross-sections are each equal to the geometrical cross-section $\pi a^{2}$ (cf. extinction paradox).
} 


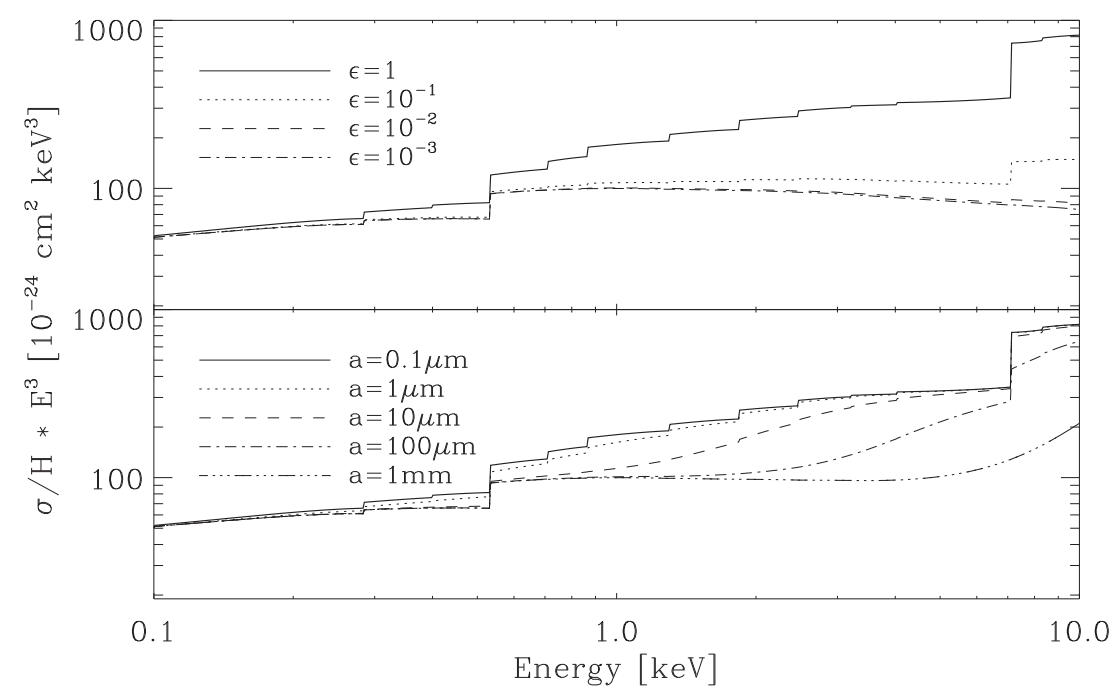

Figure 6. Effects of grain processes on the total (gas+dust) X-ray photoelectric cross-section. Top: the contribution to the X-ray opacity made by grains varies in proportion to changes in the dust:gas mass ratio, $\epsilon$, occurring as a result of dust settling. Bottom: grain growth affecting the dust opacity in a more complicated, energy-dependent manner. In both plots the gas opacity sets a basal limit.

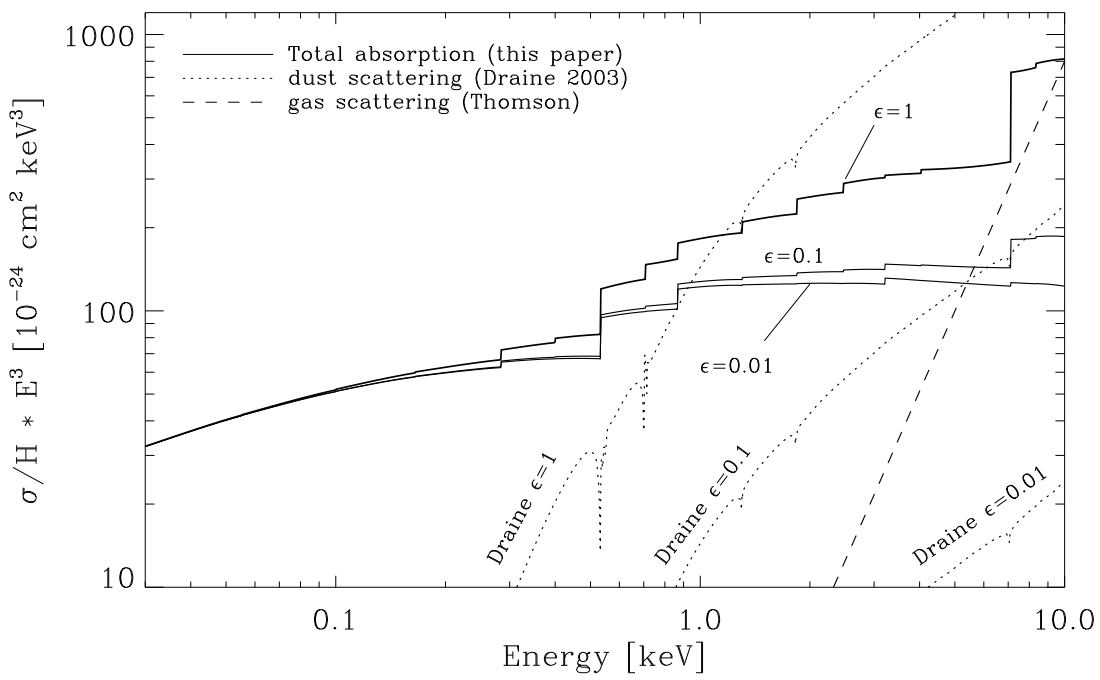

Figure 7. Comparison of the (gas+dust) photoelectric absorption (solid lines) and scattering due to gas (dashed line) and dust (dotted line), as a function of the settling parameter $\epsilon$. At $E \sim 1 \mathrm{keV}$ scattering by gas contributes negligibly to the total cross-section, whereas dust scattering is of the same order of magnitude as dust absorption. At $E_{X} \sim 10 \mathrm{keV}$ the scattering opacity is large with both gas and dust contributing significantly. Although the gas may play a role in scattering X-rays at these energies, the total photoelectric absorption is dominated by the metals in dust. Note the $E^{3}$ scaling-the (Thomson) gas scattering opacity is in fact almost independent of energy.

sorption efficiency at $E \sim 1-2 \mathrm{keV}$ is never much smaller than the scattering efficiency, the majority of X-ray photons emitted by $\mathrm{T}$ Tauri stars will have been absorbed before a significant diffuse component is generated.

\section{SUMMARY}

We have used the most recent estimates of the solar abundance to construct simple parametric fitting functions for the X-ray photoelectric cross-sections of material in T Tauri disks. By separating the medium into gas and dust components, we have shown how the X-ray opacity of $\mathrm{T}$ Tauri disk material will change as dust grows in size or settles toward the disk midplane. Here we provide a summary of our results.

1. Separate polynomial fits are presented for the photoelectric absorption cross-section of gas and dust (Tables 2 and 3, Figure 2). These fits are backward compatible with MM83.
2. Grains as large as $a \sim 1 \mu \mathrm{m}$ are required before the effect of self-blanketing is seen. This effect is most readily seen at $E \sim 1-2 \mathrm{keV}$ (Figure 3), coinciding with the peak X-ray emission of $\mathrm{T}$ Tauri systems. Due to the large fraction of small grains $(a \ll 1 \mu \mathrm{m})$, considerable grain growth is required $\left(a_{\max } \gg 1 \mu \mathrm{m}\right)$ before the MRN grain-size distribution exhibits appreciable self-blanketing at $E \sim 1 \mathrm{keV}$ (Figure 4).

3. Provided sufficiently large grains are present, the selfblanketing factor averaged over a power-law grain-size distribution is sensitive to the power-law index (Figure 5). For size distributions flatter than MRN (i.e., $p<3.5$ ), we observe a stronger ensemble-averaged self-blanketing effect, eventually asymptoting to that of a single-sized grain population with $a \sim a_{\max }$.

4. Realistic structural and compositional inhomogeneities of grains will change the self-blanketing results. 
Non-spherical grains will self-blanket less effectively. The layering of grain mantles may either increase or decrease the self-blanketing factor, depending upon the composition and order of these layers.

5. The degree of dust settling evident in protoplanetary disks, $\epsilon \sim 0.01$, is more than sufficient to reduce the X-ray opacity of dust to levels that are small compared to those of the gas (cf. Figure 6). The combined grain growth and settling observed in protoplanetary disks suggests that X-ray energy in T Tauri systems is preferentially injected into the gas component.

6. While both grain growth and settling reduce the X-ray cross-section, the remaining gas opacity sets a lower limit that ensures $\sigma_{\text {tot }}(E \sim 1 \mathrm{keV})$ does not drop below about $50 \%$ of the diffuse ISM value (Figure 6).

7. Further reduction of the X-ray cross-section in dust-evolved systems is contingent upon changes in the metal composition of the gas. The removal of $\mathrm{CO}$ and $\mathrm{Ne}$ as the gas disk dissipates could potentially reduce $\sigma_{\text {tot }}$ by a further factor of three.

X-ray counterparts have been identified for many protoplanetary disk systems in Orion, several of which display X-ray spectra consistent with absorption by surrounding material (e.g., COUP; Getman et al. 2005; Kastner et al. 2005). By comparing the absorption column inferred from X-rays to that determined from visual extinction (by dust), it is in principle possible to place constraints on the composition and dust-to-gas ratio of intervening disk material. Edge-on sources COUP 419 and COUP 241 are such examples, suggesting that the absorbing material is considerably deficient in dust (an order of magnitude or more relative to interstellar value).

Güdel et al. (2008) have detected bipolar X-ray jets emanating from the strongly accreting T Tauri system DG Tau. Comparison of the two jets indicates that one is probably viewed through an excess column of material $\left(N_{\mathrm{H}} \approx 2.7 \times 10^{21} \mathrm{~cm}^{-2}\right)$ due to the surrounding disk. Unfortunately, while this scenario potentially affords a differential analysis of the intervening material, the visual extinction along this line of sight is uncertain, rendering the gas-to-dust ratio determination inconclusive.

Far-ultraviolet (FUV) radiation is another important source of energy in protoplanetary disks. The transport of continuum FUV is largely controlled by dust, and as such is highly sensitive to dust evolution. In particular, as dust settles toward the disk midplane the upper parts of the disk become increasingly transparent to FUV photons, reducing the FUV opacity to X-ray levels. In contrast, the propagation of X-rays is impeded almost entirely by gas after only a modest degree of dust evolution $(\epsilon \sim 0.1)$. The deposition of X-rays with energies typical of the steady coronal emission from T Tauri stars $(E \sim 1-2 \mathrm{keV})$ will be deposited in the upper layers of the disk- the so-called warm molecular layer. In the models of Aikawa \& Nomura (2006), the warm layer of gaseous $\mathrm{CO}$ at $R=200 \mathrm{AU}$ has a typical vertical column density of $N_{Z}(\mathrm{CO}) \sim 10^{18} \mathrm{~cm}^{-2}$. We expect this layer to be very optically thick, $\tau_{1 \mathrm{keV}} \sim 100$, when viewed at the small grazing angles $\left(\sim 5^{\circ}\right)$ appropriate for impinging stellar X-rays. The impenetrability of this layer is mitigated somewhat at higher energies $(E \sim 10 \mathrm{keV})$ where the photoelectric cross-section becomes small compared to the scattering cross-section. In this case, Thomson scattering by gas can be an effective means of disk penetration (Igea \& Glassgold 1999).

T.B. and E.B. gratefully acknowledge funding by NASA under grant NN08 AH23G from the ATFP and SSO programs.

\section{APPENDIX \\ DERIVATION OF DUST SELF-BLANKETING FACTOR $f_{b}(E)$}

A single interstellar dust grain of sufficient size may become optically thick to X-rays. In such a case the interior atoms see a reduced flux of $\mathrm{X}$-rays relative to the atoms on the surface of the grain. The total absorptive efficacy of the dust grain is therefore less than that of an equal mass of compositionally identical gas. This effect is referred to as self-blanketing, and to calculate its magnitude we must consider the dust grain as a radiative transfer problem by itself (albeit a highly simplified one). In this appendix, we consider the self-blanketing of spherical dust grains - not because the sphere is a physically sensible model for interstellar grains but because the sphere is the most selfblanketing geometry (under isotropic illumination). The selfblanketing effect of real (i.e., non-spherical) grains will be less than that presented here. We adopt the basic notation of W00, although we differ slightly in our approach by explicitly specifying a grain shape (sphere). The average cross-section of the constituent atoms in the solid material is given by

$$
\bar{\sigma}(E)=\frac{\Sigma_{Z} A_{Z} \beta_{Z} \sigma_{Z}(E)}{\Sigma_{Z} A_{Z} \beta_{Z}}=\frac{\sigma_{\text {dust }}}{\Sigma_{Z} A_{Z} \beta_{Z}} .
$$

The atomic abundances $A_{Z}$ and depletion factors $\beta_{Z}$ are provided in Table 1. In effect Equation (A1) simply removes the per hydrogen scaling, replacing it with per grain atom. The impingent intensity $I_{0}$ is partially absorbed by the grain, emerging with an intensity $I(x)$ depending upon the offset, $x$, between the ray and the grain center. If the grain has a number density $n$ then the ray must traverse an optical depth:

$$
\tau(x)=2 \bar{\sigma} n \sqrt{a^{2}-x^{2}} .
$$

Averaged over the geometric cross-section of the grain the average emergent intensity is

$$
\begin{aligned}
\frac{\langle I\rangle}{I_{0}} & =\frac{2}{a^{2}} \int_{0}^{a} x \exp \left(-2 \bar{\sigma} n \sqrt{a^{2}-x^{2}}\right) d x \\
& =\frac{2}{\tau_{0}^{2}}\left[1-\left(\tau_{0}+1\right) \exp \left(-\tau_{0}\right)\right] .
\end{aligned}
$$

The final solution is expressed in terms of the diametrical optical depth, $\tau_{0}=2 \bar{\sigma} n a$. Since spheres look the same from all directions Equation (A3) is already angle-averaged. The fraction of radiation removed from the beam is then simply $1-\langle I\rangle / I_{0}$. If we ignore self-blanketing and assume all the constituent atoms in the grain see the same incident flux of photons, the fraction of photons removed is $\bar{\tau}=2 / 3 \tau_{0}$. Comparing this with the self-blanketed result (Equation (A3)) yields the self-blanketing factor for a spherical grain,

$$
f_{b}(E)=\frac{3}{2} \frac{1-\frac{2}{\tau_{0}^{2}}\left[1-\left(\tau_{0}+1\right) \exp \left(-\tau_{0}\right)\right]}{\tau_{0}} .
$$

Note that a grain with a small self-blanketing effect has a large $f_{b}(E)$. In W00 the grain shape is not explicitly involved in the calculation. Rather, they characterize the grain solely by a notional optical depth, $\bar{\tau}$, and their self-blanketing factor is given as

$$
f_{b}(E)^{W}=\frac{1-\exp (-\bar{\tau})}{\bar{\tau}}=\frac{1-\exp \left(-\frac{2}{3} \tau_{0}\right)}{\tau_{0}} .
$$


In the last step, we have expressed their result using the appropriate mean optical depth for a sphere, $\bar{\tau}=2 / 3 \tau_{0}$. The difference between the W00 expression and our sphere-specific result (Equation (A4)) is less than 5\% in the range $\tau_{0}=1-10$. Both functions have the same asymptotic behavior. For most purposes the computationally simpler W00 expression given by Equation (A5) will suffice. However, for grain shapes less regular than the sphere the self-blanketing factor should be evaluated in a shape-specific manner. Grain structures with low volume filling factors will be less self-blanketing.

\section{REFERENCES}

Aikawa, Y., \& Nomura, H. 2006, ApJ, 642, 1152

Arnaud, K. A. 1996, in ASP Conf. Ser. 101, Astronomical Data Analysis Software and Systems V, ed. G. H. Jacoby \& J. Barnes (San Francisco, CA: ASP), 17

Asplund, M., Grevesse, N., \& Sauval, A. J. 2005, in ASP Conf. Ser. 336, Cosmic Abundances as Records of Stellar Evolution and Nucleosynthesis, ed. T. G. Barnes, III \& F. N. Bash (San Francisco, CA: ASP), 25

Asplund, M., Grevesse, N., Sauval, A. J., \& Scott, P. 2009, ARA\&A, 47, 481

Balucinska-Church, M., \& McCammon, D. 1992, ApJ, 400, 699

Beckwith, S. V. W., Henning, T., \& Nakagawa, Y. 2000, in Protostars and Planets IV, ed. V. Mannings, A. P. Boss, \& S. S. Russell (Tucson, AZ: Univ. Arizona Press), 533

Brown, R. L., \& Gould, R. J. 1970, Phys. Rev. D, 1, 2252

Calvet, N., Patino, A., Magris, G. C., \& D'Alessio, P. 1991, ApJ, 380, 617

Chantler, C. T. 2000, J. Phys. Chem. Ref. Data, 29, 597

Charnley, S. B., Rodgers, S. D., \& Ehrenfreund, P. 2001, A\&A, 378, 1024

D’Alessio, P., Calvet, N., Hartmann, L., Franco-Hernández, R., \& Servín, H. 2006, ApJ, 638, 314

Draine, B. T. 2003, ApJ, 598, 1026

Dullemond, C. P., \& Dominik, C. 2004, A\&A, 421, 1075

Dullemond, C. P., \& Dominik, C. 2008, A\&A, 487, 205

Ercolano, B., Clarke, C. J., \& Drake, J. J. 2009, ApJ, 699, 1639

Ercolano, B., Drake, J. J., Raymond, J. C., \& Clarke, C. C. 2008, ApJ, 688, 398

Feigelson, E. D., Getman, K., Townsley, L., et al. 2005, ApJS, 160, 379

Fireman, E. L. 1974, ApJ, 187, 57
Freeman, P., Doe, S., \& Siemiginowska, A. 2001, Proc. SPIE, 4477, 76

Furlan, E., Hartmann, L., Calvet, N., et al. 2006, ApJS, 165, 568

Getman, K. V., Flaccomio, E., Broos, P. S., et al. 2005, ApJS, 160, 319

Glassgold, A. E., Najita, J., \& Igea, J. 2004, ApJ, 615, 972

Glassgold, A. E., Najita, J. R., \& Igea, J. 2007, ApJ, 656, 515

Güdel, M., Skinner, S. L., Audard, M., Briggs, K. R., \& Cabrit, S. 2008, A\&A, 478,797

Houck, J. C., \& Denicola, L. A. 2000, in ASP Conf. Ser. 216, Astronomical Data Analysis Software and Systems IX, ed. N. Manset, C. Veillet, \& D. Crabtree (San Francisco, CA: ASP), 591

Igea, J., \& Glassgold, A. E. 1999, ApJ, 518, 848

Kastner, J. H., Franz, G., Grosso, N., et al. 2005, ApJS, 160, 511

Lee, J. C., Xiang, J., Ravel, B., Kortright, J., \& Flanagan, K. 2009, ApJ, 702, 970

Lommen, D., Maddison, S. T., Wright, C. M., et al. 2009, A\&A, 495, 869

Maloney, P. R., Hollenbach, D. J., \& Tielens, A. G. G. M. 1996, ApJ, 466, 561

Mathis, J. S., \& Lee, C. 1991, ApJ, 376, 490

Mathis, J. S., Rumpl, W., \& Nordsieck, K. H. 1977, ApJ, 217, 425

Morrison, R., \& McCammon, D. 1983, ApJ, 270, 119

Nomura, H., Aikawa, Y., Tsujimoto, M., Nakagawa, Y., \& Millar, T. J. 2007, ApJ, 661,334

Overbeck, J. W. 1965, ApJ, 141, 864

Owen, J. E., Ercolano, B., Clarke, C. J., \& Alexander, R. D. 2010, MNRAS, 401, 1415

Pascucci, I., Hollenbach, D., Najita, J., et al. 2007, ApJ, 663, 383

Predehl, P., \& Schmitt, J. H. M. M. 1995, A\&A, 293, 889

Rolf, D. P. 1983, Nature, 302, 46

Smith, R. K., \& Dwek, E. 1998, ApJ, 503, 831

Sofia, U. J. 2004, in ASP Conf. Ser. 309, Astrophysics of Dust, ed. A. N. Witt, G. C. Clayton, \& B. T. Draine (San Francisco, CA: ASP), 393

Thi, W. F., van Dishoeck, E. F., Blake, G. A., et al. 2001, ApJ, 561, 1074

Throop, H. B., Bally, J., Esposito, L. W., \& McCaughrean, M. J. 2001, Science, 292, 1686

van de Hulst, H. C. (ed.) 1957, Light Scattering by Small Particles (New York: Dover Publications)

Weidenschilling, S. J., \& Cuzzi, J. N. 1993, in Protostars and Planets III, ed. E. H. Levy \& J. I. Lunine (Tucson, AZ: Univ. Arizona Press), 1031

Weingartner, J. C., \& Draine, B. T. 2001, ApJ, 548, 296

Wilms, J., Allen, A., \& McCray, R. 2000, ApJ, 542, 914

Wilner, D. J., D’Alessio, P., Calvet, N., Claussen, M. J., \& Hartmann, L. 2005, ApJ, 626, L109 